\title{
Como gestores planejam seu comportamento nas redes sociais online
}

A utilização crescente das redes sociais online, a interação dinâmica que estas proporcionam, a manifestação de comportamentos e imagem das pessoas que estão conectadas às mesmas, são apresentadas na pesquisa com o objetivo de compreender como os gestores planejam seu comportamento nas redes sociais online, com base na teoria do comportamento planejado do Ajzen (1991). O estudo, de abordagem qualitativa, desenvolvido por meio de entrevistas de cunho exploratório teve a amostra de 09 gestores, com base na técnica do incidente crítico, para identificar os atributos específicos de comportamento, considerando os desdobramentos positivos e negativos, e a análise de fatos importantes relacionados ao planejamento comportamental dos gestores nas redes sociais, em específico o Facebook. Os resultados encontrados mostraram que o modelo teórico de Ajzen (1991) aplica-se de forma significativa à explicação do comportamento planejado dos gestores. A principal observação recai sobre a ação do construto controle do comportamento percebido, como agente moderador e corretor das ações de comportamento planejado dos gestores. Foi constatado, também, que os gestores planejam o seu comportamento, muitas das vezes, sem levar em consideração os seus valores, que de acordo com o modelo teórico, está atrelado às normas subjetivas do indivíduo, porém ficou evidenciado um maior cuidado com a sua imagem refletida em suas ações comportamentais no Facebook, considerando que no ambiente profissional, os mesmos ocupam cargos que são observados por sua equipe e demais profissionais.

Palavras-chave: Redes sociais online; Comportamento planejado; Gestores.

\section{How managers plan your behavior on online social networks}

The increasing use of online social networks, the dynamic interaction they provide, the manifestation of behaviors and the image of people who are connected to the same, are presented in the research in order to understand how managers plan their behavior in online social networks, based on theory of planned behavior of Ajzen (1991). The qualitative study, developed through interviews of an exploratory nature, had the sample of 09 managers, based on the technique of the critical incident, to identify the specific attributes of behavior, considering the positive and negative unfoldings, and the analysis of facts related to behaviora planning of managers in social networks, specifically Facebook. The results showed that Ajzen's theoretical model (1991) applies in a significant way to the explanation of the planned behavior of the managers. The main observation rests on the action of the control construct of the perceived behavior, as agent moderator and corrector of the behavior actions of the managers. It was also observed that managers plan their behavior, many times, without taking into account their values, which according to the theoretical model, is linked to the subjective norms of the individual, but it was evidenced a greater care with their image reflected in their behavioral actions on Facebook, considering that in the professional environment, they occupy positions that are observed by their team and other professionals.

Keywords: Online social networks; Planned behavior; Managers.

\section{Topic: Recursos Humanos}

Reviewed anonymously in the process of blind peer.

Leonardo Quintas Rocha

Faculdade de Tecnologia, Brasil

http://lattes.cnpq.br/6311570691842760

larocha@hotmail.com

\section{Ariana Marchezi de Souza}

Universidade Federal do Espírito Santo, Brasil

http://lattes.cnpq.br/9953699667636026

arianamds@yahooo.com.br

\section{Alexandre dos Santos Cerqueira}

Universidade Federal do Espírito Santo, Brasil

http://lattes.cnpq.br/0295365714362377

alexandrecerqueira1971@yahoo.com.br

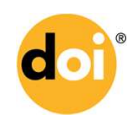

DOI: 10.6008/SPC2179-684X.2017.001.0010
Received: 15/01/2017

Approved: 15/03/2017

Miguel Carlos Ramos Dumer

Universidade Federal do Espírito Santo, Brasil

http://lattes.cnpq.br/5216143663685024

prof.migueldumer@gmail.com
Referencing this:

ROCHA, L. Q.; SOUZA, A. M.; CERQUEIRA, A. S.; DUMER, M. C. R. Como gestores planejam seu comportamento nas redes sociais online. Revista Brasileira de Administração Científica, v.8, n.1, p.118-130, 2017. DOI: http://doi.org/10.6008/SPC2179-684X.2017.001.0010 


\section{INTRODUÇÃO}

As redes sociais online (RSO) atraem pessoas do mundo inteiro, tanto no âmbito pessoal quanto profissional. A participação e o compartilhamento de informações se tornam uma forma dinâmica de interação, mesmo considerando que estas redes possam representar riscos à privacidade por estarem inseridas em uma esfera pública, que é a internet (O'BRIEN et al., 2012; KWON et al., 2010; CHAFFEY et al., 2009; ROSENBLUM, 2007).

Neste contexto, a literatura tem abordado o uso das RSO como forma de interação, comunicação e gestão de imagem, além de compreender o uso dessa ferramenta e suas influências (O'BRIEN et al., 2012; PIMMER et al., 2012; NADKARNI et al., 2012). Por exemplo, Baker et al. (2010) buscou compreender a dinâmica da usabilidade e o comportamento do indivíduo nas RSO, investigando as influências do envolvimento de adolescentes com as RSO, por meio da teoria do comportamento planejado (TCP). Ainda no âmbito comportamental, Al-Debei et al. (2013) estudou as intenções de participação dos indivíduos e seus comportamentos no Facebook, com foco nos aspectos sociais que podem determinar o uso contínuo da ferramenta. Porém, poucos estudos têm buscado compreender o comportamento do indivíduo nas RSO no aspecto profissional (RANIERI et al., 2012; TURVEY, 2012; MCEACHERN, 2011).

Ollier-Malaterre et al. (2012) realizou um estudo para identificar como os profissionais gerenciam sua identidade pessoal e profissional nas RSO, por meio de um modelo de identificação comportamental, mas não preencheram a lacuna sobre como os indivíduos planejam o seu comportamento nas RSO. A despeito desses estudos (BAKER et al., 2010; GOLWAL et al., 2012; O'BRIEN et al., 2012; PIMMER et al., 2012; NADKARNI et al., 2012, OLLIER-MALATERRE et al., 2012), no sentido de contribuir para o entendimento do comportamento do indivíduo em aspectos sociais, pessoais e profissionais nas RSO, a presente pesquisa tem o propósito de compreender como os gestores planejam seu comportamento nessas redes sociais, com base na teoria do comportamento planejado de Ajzen (1991).

A Teoria do Comportamento Planejado (TCP) de Ajzen (1991) é utilizada para explicação de comportamento planejado em distintas áreas, como empreendedorismo, finanças, saúde, educação, dentre outras (PERRY et al., 2013; XIAO et al., 2011; PICKETT et al., 2013; CÔTÉ et al., 2012; PARK et al., 2013), porém ainda não se percebeu na literatura a aplicação da mesma na compreensão do comportamento dos gestores nas RSO. Desta forma, esse estudo se propõe a preencher esta lacuna.

Considerando a massiva utilização das RSO, por exemplo, o Facebook, por indivíduos ao redor do mundo e em áreas pessoais e profissionais (GOLWAL et al., 2012; LAMPE et al., 2006; GROSS et al., 2005; STUTZMAN, 2006), faz-se necessário entender como é manifestado o comportamento planejado de um gestor nas RSO. No ambiente organizacional, a pesquisa desenvolvida pode auxiliar o gestor a entender e analisar os precedentes de um comportamento manifestado de forma planejada, e então auxiliá-lo a manifestar o seu próprio comportamento de modo mais coerente e proveitoso para sua carreira.

Este artigo está baseado na literatura sobre a relevância e o processo de interação nas RSO e em seguida, versa sobre a teoria do comportamento planejado, aplicada no campo de estudo das RSO. A metodologia qualitativa foi utilizada para testar esses pressupostos teóricos, por meio de entrevistas de 
cunho exploratório com base no método do incidente crítico. Sucede-se a apresentação e a discussão dos resultados. $\mathrm{O}$ estudo foi encerrado com as conclusões, sugestões práticas e acadêmicas e as limitações da pesquisa.

\section{REVISÃO TEÓRICA}

\section{As redes sociais online}

O serviço das RSO tem características de serviços baseados na web, permitindo ao indivíduo criar o seu perfil com abertura para divulgação de informações, imagens e vídeos com outros usuários conectados a sua rede (KWON et al., 2010; WILCOX et al., 2013). As RSO são ferramentas que trazem utilidade para educação informal, identidade coletiva, relações sociais, construção e fortalecimento de comunidades e o bem-estar individual, como descreve Hwang (2013), em sua pesquisa dos benefícios e desafios das RSO. Ainda neste contexto, Wilcox et al. (2013) e Darvell et al. (2011) apresentam as RSO como estímulo motivacional, atribuindo a sua interação como antecedente para atender necessidades sociais, autoexpressão, bem como tem efeito positivo no bem-estar das pessoas.

As RSO vêm se tornando um atrativo como meio de comunicação e interação entre os indivíduos (WILCOX et al., 2013) e a crescente utilização destas decorre da necessidade de compartilhar opiniões, informações e socializar (O'BRIEN et al., 2012; KWON et al., 2010). A participação nas RSO gera um encontro de diversidade cultural, social e intelectual que tende a explicar como ocorre a interação com outras pessoas por interesses semelhantes (KWON et al., 2010), além de se tornar uma fonte de informações e uma ferramenta complementar na gestão da imagem de um indivíduo (MCEACHERN, 2011). Dado este contexto, uma quantidade expressiva de informações pessoais são divulgadas nas RSO, por meio do recurso timeline do Facebook, por exemplo, o qual exibe, em forma de exposição cronológica, as informações publicadas pelo indivíduo (OLLIER-MALATERRE et al., 2012). Essa exposição tende a refletir o comportamento adotado por um indivíduo no ambiente das RSO, comportamento este que pode levar o indivíduo a ter de planejar seu comportamento por questões profissionais (OLLIER-MALATERRE et al., 2012).

As RSO trazem a interação do indivíduo com pessoas, que previamente são aceitas em sua rede de relacionamentos, e também pessoas que não fazem parte de sua rede, mas que podem apenas visualizar as informações básicas publicadas (OLLIER-MALATERRE et al., 2012). Deste modo, o comportamento manifestado na RSO tende a ser construído de modo planejado, segundo o modelo proposto por Ajzen (1991), que trata o comportamento como a manifestação, observável, das intenções de um indivíduo.

A participação nas RSO, por diversos perfis que ocupam o cargo de gestor, sugere o estudo de averiguação da existência de planejamento de comportamento nas redes sociais online por parte de indivíduos no cargo de gestor (OLLIER-MALATERRE et al., 2012). A gestão de comportamento e imagem no uso das RSO tornam um exercício constante diante da pressão social existente nas próprias RSO (OLLIERMALATERRE et al., 2012), impulsionadas pelas atitudes relacionadas ao comportamento e a sensibilidade de controlar o comportamento percebido, de acordo como o modelo de Ajzen (1991). No entanto, poucos estudos abordam a existência de planejamento de comportamento nas RSO, por parte dos indivíduos com 
cargos de gestão nas organizações, utilizando como pano de fundo a teoria do comportamento planejado de Ajzen (1991).

\section{Teoria do comportamento planejado}

A teoria do comportamento planejado é um modelo originado da psicologia, que avalia e prevê as intenções e comportamentos do indivíduo em situações que o mesmo pode não apresentar condições de controle de seu próprio comportamento (AJZEN, 1991; AL-DEBEl et al., 2013). Esta teoria aborda como ponto central as intenções do comportamento do indivíduo que serão determinantes para execução do comportamento (AL-DEBEl et al., 2013; DARVELL et al., 2011).

É possível que a previsão das intenções, para a efetivação de distintos tipos de comportamentos, com elevado nível de precisão, possa ser realizada a partir de atitudes relacionadas com as normas subjetivas, controle do comportamento percebido e atitudes relacionadas ao comportamento que, em conjunto, geram as intenções idealizadoras do comportamento adotado (AJZEN, 1991). Portanto, a possibilidade de realizar determinados comportamentos aumenta quando as intenções dos indivíduos são sinalizadas para executar estes comportamentos (BAKER et al., 2013).

A teoria do comportamento planejado é o modelo que mais se aproxima das intenções e do comportamento adotado por indivíduos ao se tratar de estudos sobre RSO (AL-DEBEl et al., 2013; BAKER et al., 2013; DARVELL et al., 2011; PELLING et al., 2009). O uso da teoria do comportamento planejado neste estudo deve-se ao fato em que as RSO, por exemplo, o Facebook, tem como propósito atender as necessidades sociais de seus usuários, em vez de suas necessidades nos aspectos profissionais (AL-DEBEl et al., 2013). Desta forma, este estudo tem interesse no comportamento dos indivíduos e em sua forma de planejamento, dada as experiências vividas com o uso da tecnologia. A figura 1 apresenta o modelo de Ajzen (1991).

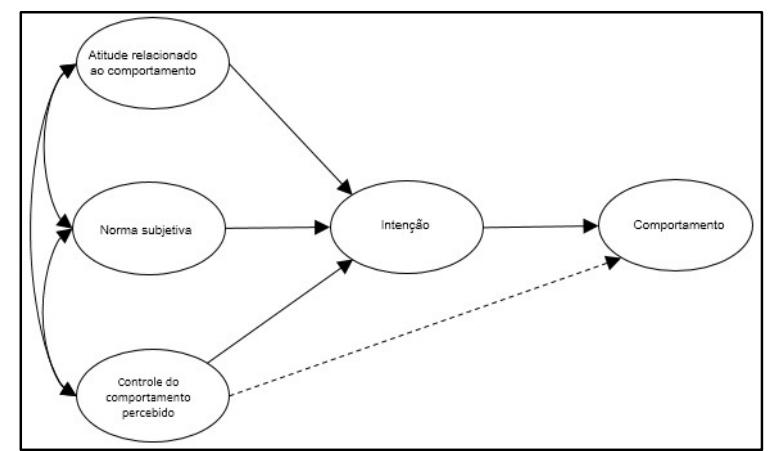

Figura 1: Teoria do comportamento planejado. Fonte: Ajzen (1991).

\section{Normas Subjetivas do Indivíduo}

Para compreender a teoria do comportamento planejado (TCP) é necessário entender os componentes que antecedem a transformação das intenções do indivíduo no comportamento manifestado (AJZEN, 1991). O indivíduo pertinente a este processo é descrito por Ajzen (1991) como um ser dotado de características, valores e questões pertinentes a sua cultura, dentre outras características. 
A observação de como o comportamento de um indivíduo é influenciado pelo desejo de agir, conforme seus referenciais (AL-DEBEl et al., 2013), é analisado no contexto das RSO sob a ótica de como os gestores reagem a pressões sociais e decidem pela utilização das RSO em seu cotidiano, ou não. Após a tomada de decisão em participar das RSO, o presente estudo analisa como esse conjunto de crenças normativas age sobre o comportamento planejado de gestores nessas redes, considerando as observações relatadas em estudos anteriores (AJZEN, 1991; AL-DEBEl et al., 2013; BAKER et al., 2013; DARVELL et al., 2011; PELLING et al., 2009; OLLIER-MALATERRE et al., 2012).

\section{Atitude Relacionada ao Comportamento}

Para Ajzen (1991), a atitude relacionada ao comportamento refere-se ao grau que uma pessoa tem de avaliar como favorável, ou não favorável, o comportamento em questão a ser executado. De modo geral, atitudes relacionadas ao comportamento fazem menção à construção de avaliações mais amplas, no contexto externo ao indivíduo, de modo que o mesmo possa examinar o comportamento global, tanto positivamente quanto negativamente (AL-DEBEl et al., 2013; BAKER et al., 2010). Nas RSO, essa identificação de conteúdo como favorável ou não, pode gerar pressupostos para os gestores avaliarem sua conduta comportamental ao utilizarem essas ferramentas, como o Facebook (OLLIER-MALATERRE et al., 2012; AJZEN, 1991).

\section{Controle do Comportamento Percebido}

O controle do comportamento percebido está relacionado à capacidade e à percepção que o indivíduo tem em realizar um determinado comportamento, mediante a normatização social aplicada ao mesmo (AJZEN, 1991), conforme recursos e oportunidades disponíveis (HSU, 2013; AL-DEBEl et al., 2013). As RSO são campos de estudo onde os indivíduos, de uma forma geral, manifestam seus comportamentos e interagem com outros (AL-DEBEl et al., 2013; OLLIER-MALATERRE et al., 2012; GOLWAL, 2012). Ao considerar o modelo de TCP proposto por Ajzen (1991), pode-se perceber que a normativa de conduta nessas RSO são avaliadas por cada indivíduo, com base em seu conjunto de crenças e após a análise do indivíduo é que o comportamento planejado será manifestado, relação essa que o presente estudo também se propõe a analisar.

\section{Intenção}

A intenção é a sinalização da disponibilidade de uma pessoa a realizar um determinado comportamento, como conceitua Ajzen (1991) na TCP, também considerada como o antecedente imediato do comportamento. Nesse estudo, essas intenções foram observadas no comportamento de gestores nas RSO. Na TCP, a intenção é a resultante da interação de três itens: atitude em relação ao comportamento, norma subjetiva e controle comportamental percebido (AJZEN, 1991; BAKER et al., 2010; DARVELL et al., 2011). 


\section{Comportamento}

A TCP tem a capacidade preditiva de compreender a manifestação comportamental de um indivíduo de um modo mais próximo à realidade (AJZEN, 1991). O comportamento manifestado é uma resultante da intenção de um indivíduo em realizá-lo (AJZEN, 1991). No contexto das RSO, o estudo utiliza o modelo de TCP (AJZEN, 1991) para analisar como gestores planejam o seu comportamento. O comportamento manifestado nas RSO pode proporcionar ao gestor uma maior interação nas plataformas sociais, intelectuais e profissionais, ao ampliar sua interação com indivíduos que não estão presentes diariamente em seu cotidiano (ZAKARIA et al., 2010).

\section{METODOLOGIA}

Com o objetivo de investigar como os gestores planejam seu comportamento nas redes sociais online, realizou-se estudo de abordagem qualitativa, utilizando a técnica do incidente crítico (FLANAGAN, 1973) para identificar os atributos específicos de comportamento e analisar fatos importantes relacionados ao planejamento comportamental dos gestores nas RSO. Bitner et al. (1990) explicaram que um incidente crítico contribui significativamente, tanto de forma positiva quanto negativa, para uma atividade ou fenômeno.

A pesquisa que utiliza a técnica do incidente crítico (TIC) permite que os entrevistados expressem suas percepções e comportamentos, podendo classificá-los em fatores satisfatórios ou insatisfatórios, como relatou Johnston (1995) em seu estudo. Na abordagem de Gremler (2004), a TIC tem sido utilizada em cenário de serviços, com objetivo de melhor investigar a satisfação ou insatisfação no uso dos mesmos.

Nesta pesquisa, a TIC foi inserida no contexto comportamental, com o propósito de analisar a adequação do modelo de Ajzen (1991) à manifestação do comportamento planejado de gestores nas RSO. Essa análise parte de um entendimento inicial do motivo pela qual esses gestores adotam a RSO e posteriormente como planejam seu comportamento, tomando por base acontecimentos que tenham proporcionado experiências negativas e positivas.

Neste contexto, Flanagan (1973) define que a TIC toma como base um conjunto de procedimentos para a coleta de observações do comportamento humano. Logo, cada incidente crítico ocorrido nas RSO com os gestores, aponta a um comportamento planejado distinto, o que contribui para compreensão da adequação da TCP (AJZEN, 1991) nesse campo. Os critérios utilizados para definição do perfil dos entrevistados foram:

Indivíduos com cargo de gestão, atuantes no mercado no mínimo por dois anos, por se considerar um tempo relevante para o desenvolvimento do perfil de liderança, bem como ter tido a oportunidade de passar por experiências positivas e negativas nas RSO durante esse tempo;

Como campo de estudo a RSO Facebook foi escolhida, dado que a mesma possui um importante crescimento e aceitação desde o seu desenvolvimento, no ciberespaço (GOLWAL et al., 2012; LAMPE et al., 2006; GROSS et al., 2005; STUTZMAN, 2006);

Gestores que não possuem a obrigatoriedade de ter perfis nas RSO e que tenham em seu grupo de contatos, pessoas de seu convívio pessoal e profissional; 
Foram considerados somente os gestores que fazem a administração de sua conta no Facebook, para evitar qualquer tipo de desvio comportamental, por parte da gestão de um terceiro;

Gestores que possuem contas para distintos perfis nas RSO não são relevantes a pesquisa, pois tendem a ter comportamentos distintos nos perfis (OLLIER-MALATERRE et al., 2012).

As entrevistas com os gestores, num total de 09 entrevistados para a amostra, foram realizadas por meio de um roteiro semiestruturado, com questões apoiadas na utilização das RSO de acordo com a categorização do modelo de Ajzen (1991). As mesmas foram realizadas nos locais de trabalho dos entrevistados, porém em salas particulares, de modo que o mesmo não se sentisse inibido e, também em ambientes neutros e externos à organização dos entrevistados.

Os entrevistados tiveram sua identidade preservada, bem como o nome da organização em que o mesmo atua, por uma questão de sigilo de informação e preservação da imagem do entrevistado. Num primeiro momento, os entrevistados foram arguidos sobre os motivos que o levaram a adotar a criação de uma RSO, no caso o Facebook, assim como a utilização da ferramenta no seu cotidiano.

$\mathrm{Na}$ sequência, as entrevistas foram conduzidas de modo que os entrevistados relatassem detalhadamente, o seu ingresso na RSO Facebook, a forma e a frequência de utilização, as situações pelas quais passaram, no Facebook, e tiveram como resultantes experiências positivas e negativas, ou que tenham tido conhecimento de pessoas próximas ao seu convívio, com perfil profissional semelhante e tenham passado por experiências comportamentais, no Facebook, com desdobramentos positivos e negativos.

A análise dessas entrevistas foi realizada por meio da técnica de análise de conteúdo (BARDIN, 1977), associando o método TIC (FLANAGAN, 1973) ao modelo de TCP proposto por Ajzen (1991), no intuito de compreender como os gestores entrevistados planejam seu comportamento nas RSO. O quadro 1 sintetiza o perfil dos gestores conforme a idade, sexo, estado civil, tempo no cargo de gestão e tempo de existência do perfil no Facebook.

Quadro 1: Perfil da amostra de entrevistados.

\begin{tabular}{|c|c|c|c|c|c|c|}
\hline Código & Idade & Sexo & $\begin{array}{c}\text { Estado } \\
\text { Civil }\end{array}$ & Cargo & $\begin{array}{c}\text { Tempo } \\
\text { no cargo }\end{array}$ & Existência de perfil no Facebook \\
\hline Ger01 & 41 anos & $\mathrm{M}$ & Casado & Gerente de $\mathrm{TI}$ & 10 anos & 4 anos \\
\hline Ger02 & 36 anos & $\mathrm{F}$ & Casada & Gerente de Projetos & 3 anos & 2 anos \\
\hline Ger03 & 43 anos & $\mathrm{F}$ & Solteira & $\begin{array}{l}\text { Gerente de } \\
\text { Planejamento }\end{array}$ & 7 anos & 3 anos \\
\hline Ger04 & 32 anos & $\mathrm{F}$ & Solteira & $\begin{array}{l}\text { Coordenadora } \\
\text { Pedagógica }\end{array}$ & 3 anos & 3 anos \\
\hline Ger05 & 47 anos & $\mathrm{F}$ & Casada & Gerente de RH & 2,5 anos & 3 anos \\
\hline Ger06 & 40 anos & $\mathrm{M}$ & Casado & $\begin{array}{l}\text { Coordenador } \\
\text { Pedagógico }\end{array}$ & 4 anos & 2 anos \\
\hline Ger07 & 35 anos & $\mathrm{F}$ & Casada & Diretora de Negócios & 7 anos & 2 anos \\
\hline Ger08 & 35 anos & $\mathrm{M}$ & Solteiro & Gerente de Projetos & 4 anos & 3 anos \\
\hline Ger09 & 33 anos & $\mathrm{M}$ & Solteiro & Gerente de Negócios & 2 anos & 3 anos \\
\hline
\end{tabular}

\section{RESULTADOS}

\section{Normas subjetivas}

O conjunto de crenças normativas sobre os referenciais do indivíduo que é classificado como normas subjetivas, onde cada crença normativa é analisada por uma motivação referenciada (AJZEN, 1991). Baker et al. (2013) ressalta que a norma subjetiva está diretamente relacionada à percepção da pressão social por 
meio de características relevantes, para realizar ou não o comportamento do indivíduo. Na pesquisa de Xiao et al. (2011), a utilização de cartão de crédito por estudantes universitários foi desenvolvida utilizando a TCP de Ajzen (1991), e se observou que a norma subjetiva dos pais influencia significativamente nos comportamentos de seus filhos no que tange questões financeiras.

Durante o processo de adequação dos códigos gerados ao modelo teórico proposto por Ajzen (1991), com base nos entrevistados, foi possível identificar características semelhantes, como a restrição quanto à publicação e a análise do conteúdo das mesmas. A preocupação em manifestar comportamentos, de acordo com os gestores, é em grande parte atrelado ao cuidado de sua imagem, pois eles acreditam que situações complexas ou constrangedoras, relacionadas ao seu perfil no Facebook, possam denegrir a sua imagem profissional. Dentre os gestores entrevistados, foram identificados alguns com características antagônicas (GER 06 e GER 07), em relação à apreciação da exposição de imagem, porém continuam presentes e com manifestações no Facebook, independente da visão crítica que fazem quanto a RSO. No quadro 2, destacamse as ponderações de alguns entrevistados.

Quadro 2: Normas Subjetivas: fragmentos do conteúdo das entrevistas.

“...Basicamente, qualquer coisa que eu seja citada, passa pela minha autorização, eu não permito que seja publicado nada sem
que eu veja o que está sendo publicado, mas isso acontece quando eu sou citada..." (GER05, $\mathbf{4 7}$ Anos, Gerente de RH.)
“...Um monte de coisa maluca que você acaba observando no facebook e sinceramente acho que é um dos motivos pelo qual eu
não estou acessando tanto [...] É muita exposição" (GER06, $\mathbf{4 0}$ anos, Coordenador Pedagógico)
“...Então eu não fico em cima do muro, eu sempre expresso ali o que eu estou achando mesmo [...] Eu gosto disso, eu sempre fui
assim, nunca tive preocupação de me expor e como gestora..." (GER07, 35 anos, Diretora de Negócios)

\section{Atitude relacionada ao comportamento}

A percepção do indivíduo ao observar as atitudes externas no campo da RSO Facebook tende a ser um fator relevante, dado que a sua manifestação comportamental pode ser utilizada e visualizada como conteúdo para atrair pessoas, assim como para a interação com estas, no tocante a publicações de conteúdo específicos (GOLWAL, 2012). Conforme os relatos dos gestores, a atitude relacionada ao comportamento ficou evidente ao apresentar situações na RSO Facebook, onde o gestor expõe sua percepção ao observar as atitudes de pessoas pertencentes ao seu grupo no Facebook.

Os gestores de modo geral expressam o quanto a ferramenta poderia ser melhor utilizada pelos usuários das RSO. Enfatizam, ainda, que a ferramenta é de fácil acesso, utilizado em um ambiente popularmente conhecido, que é a internet, e alertam para as atenções que as pessoas devem ter ao publicar uma informação e nas consequências que esse comportamento pode trazer, como se observa nos fragmentos de entrevista apresentados no quadro 3.

Quadro 3: Atitude Relacionada ao Comportamento: fragmentos do conteúdo das entrevistas.

\footnotetext{
“...As pessoas não tem a malícia, a qualificação técnica pra colocar os filtros, muitas vezes usam de maneira inadequada, não sabem até onde a informação pode chegar, não tem ideia da dimensão e as pessoas divulgam as questões pessoais, expõe fragilidade emocional, fragilidade financeira..." (GER01, 41 anos, Gerente de TI).

"...Isso tem preocupado bastante né, eu acho que cada vez mais as pessoas tem perdido a noção e o limite entre o que é o público e privado né, esse é o grande ponto, a gente se depara com fotos, depoimentos, enfim, com situações que por vezes eu chego a considerar constrangedor né, considerando que isso possa vir de um colega de trabalho de alguém da família ou de um amigo, né, de uma rede de amigos mesmo..." (GER05, 47 anos, Gerente de Recursos Humanos).

“...As pessoas tem a necessidade de autoafirmação, publicam informações do que estão fazendo, pra onde estão indo, ainda mais quando estão ostentando algo, ou seja, utilizam o Facebook como forma de se mostrar, ao invés de aproveitar a facilidade de interação com as pessoas de forma online, na qual a ferramenta se propõe..." (GER08, 35 anos, Gerente de Projetos).
} 


\section{Controle do comportamento percebido}

Em seu estudo, Ajzen (1991) relatou que o controle do comportamento percebido é determinado pelo conjunto de crenças do indivíduo, sendo que essas crenças se destacam sob a presença de fatores que podem facilitar ou dificultar o desempenho do comportamento. No estudo de Côtéet al. (2012), controle do comportamento percebido também contribuiu para prever a intenção dos enfermeiros no âmbito de tomada de decisão clínica.

Dentre a amostra de gestores estudada, observou-se que o controle do comportamento percebido é a variável de maior impacto sobre o comportamento planejado dos gestores. A recorrência de uma autoanálise antes de manifestar qualquer tipo de comportamento no Facebook, foi citada por esses gestores, dado que os mesmos ocupam cargos de destaque e coordenam uma equipe, a qual um bom modelo e exemplo precisam ser propagados.

Independente da imagem que os gestores transpareçam à sua equipe, estes também precisam tratála e apresentá-la para os demais colegas, da mesma ou de outra organização. A sua reputação profissional precisa ser gerida também em suas redes sociais, caso as tenham, pois mesmo que o ambiente não seja um espaço formal para análise de perfis, as informações ali geradas podem ser propagadas em seu meio profissional. O comportamento com base nos parâmetros aceitos pelo mercado pode influenciar na carreira de forma positiva ou negativa, conforme explicitado pelos gerentes 05, 07 e 09.

Quadro 4: Controle do Comportamento Percebido: fragmentos do conteúdo das entrevistas.

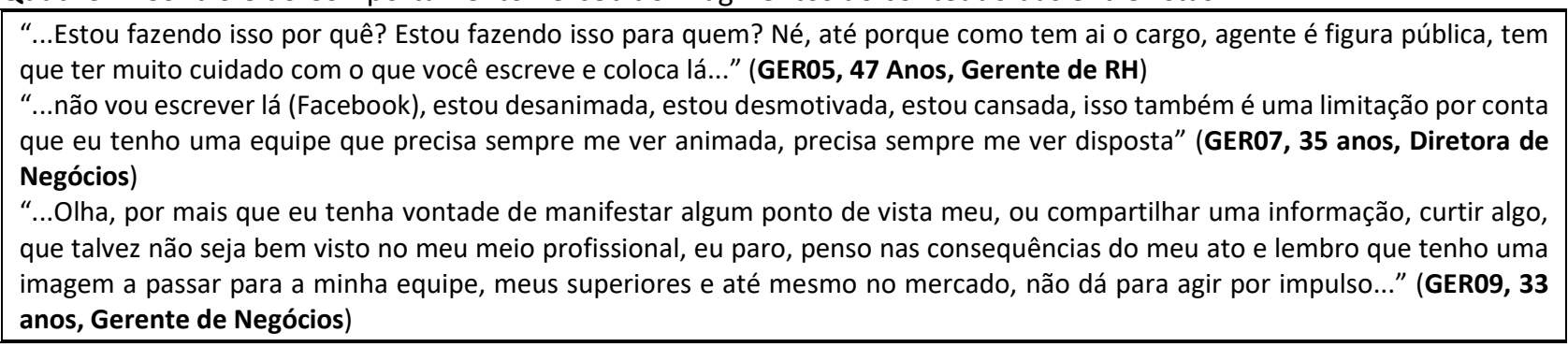

\section{Intenções}

Na RSO Facebook, a teoria do comportamento planejado é analisada pelo presente estudo, no intuito de compreender como gestores constroem suas intenções, antes de manifestar a realização das mesmas. A intenção de adotar o uso da RSO Facebook tem como base três antecedentes propostos por Ajzen (1991): as atitudes relacionadas ao comportamento, a norma subjetiva e o controle do comportamento percebido. Conforme a TCP, pode-se constatar nos depoimentos dos gestores que há uma intenção ao criar um perfil no Facebook, ou até mesmo, uma intenção para realizar um comportamento específico. Intenção essa que configura seu planejamento para manifestar o comportamento.

Os gestores entrevistados, de modo geral, abordaram o motivo pelo qual adotaram a ferramenta, atrelados ao fato de conhecer seu propósito, interagir com as pessoas, além de situações específicas, como se pode observar no quadro 5. Diante deste cenário, os entrevistados não criaram o seu perfil no Facebook de forma impulsiva. 
Quadro 5: Intenções: fragmentos do conteúdo das entrevistas.

"...Criei minha conta no Facebook, para facilitar a forma de comunicação com meus amigos e também para aumentar meu
networking ..." (GER02, $\mathbf{3 6}$ anos, Gerente de Projetos).
"...Utilizo o Facebook para monitorar meu sobrinho, acompanhar suas publicações devido sua idade, pela interatividade que o
Facebook proporciona e para divulgação do meu trabalho que tenho tido sucesso..." (GER03, $\mathbf{4 3}$ anos, Gerente de Planejamento).
"...Antes de publicar alguma opinião, me policio para que essa publicação não seja mal interpretada, mas eu estou sempre
preocupada se aquilo está de acordo com o que eu sou mesmo, se eu não estou fantasiando um comportamento, se eu não estou
fantasiando alguma opinião..." (GER07, 35 anos, Diretora de Negócios).

\section{Comportamento}

O comportamento manifestado de forma planejada, pelos gestores entrevistados, foi o principal foco de estudo proposto por essa pesquisa. Os desdobramentos para compreensão sobre o planejamento comportamental foram investigados por meio de consequências positivas e negativas, resultantes de um comportamento adotado por esse gestor, ou de seu conhecimento, dado que o indivíduo que o vivenciou, possui um perfil adequado ao proposto na metodologia do artigo.

Quadro 6: Comportamento: fragmentos do conteúdo das entrevistas.

POSITIVO
“...Foi há exatamente 1 ano atrás [...] Eu comecei a fazer uma
formação e eu fiz um comentário sobre o dia em que eu estava
começando, aquela formação e é uma formação que até para
que eu tenha continuidade eu vou precisar de clientela, eu vou
precisar de indicações e pessoas, formadoras de opinião, da
minha rede, em especial 2 das pessoas, viram e fizeram
comentários, pediram informações e compartilharam, e isso
gerou um interesse maior [...] Mas de maneira nenhuma ela foi
feita com esse caráter mais promocional e acabou trazendo uma
repercussão, positiva. (GER05, 47 Anos, Gerente de RH)
“...São vários, por exemplo, por conta dessa questão, que eu
trabalho na área comercial, então eu já fui abordada várias vezes
por empresas querendo contratar o meu trabalho, contratar a
minha empresa, graças a uma rede social [...] Uma vez nós
publicamos uma campanha e foi uma ação de vendas [...] Tudo
o que você coloca ali, você tem um objetivo ou pessoal ou
profissional, não acho que é só informativo, as pessoas veem o
que você está fazendo, veem com o que você está trabalhando,
como é que você lida com a vida pessoal...” (GER07, 35 anos,
Diretora de Negócios)
Diretora de Negócios)

\section{NEGATIVO}

“... Me lembro, um profissional, um colega de profissão, diretor de recursos humanos [...], me disse que sempre gostou muito de manter o perfil no facebook, mas que perdeu esse perfil numa conversa com a esposa, porque ele foi a um determinado evento e lá fizeram uma foto dele num grupo de meninas, numa pose mais informal, essa foto circulou no Facebook, a esposa dele viu, ele disse que estava num evento profissional e era um evento profissional, mas as pessoas estavam com uma postura mais informal e isso trouxe uma consequência negativa, porque ele perdeu, ela (a esposa) fez com que ele tirasse. Eu considero isso uma consequência negativa, na medida em que é um profissional da área de recursos humanos que inclusive precisa ai desse caminho, para poder acessar pessoas e porque teve a sua imagem de certa forma afetada por esse episódio..." GER05, 47 Anos, Gerente de $\mathbf{R H}$

“... Eu fiquei revoltada com a morte do menino que fez parte das manifestações em uma cidade do interior de São Paulo, eu fiquei revoltada com aquilo. Então foi um negócio que eu me expus, que eu esperava ser compreendida e não fui, porque, por exemplo, eu postei uma foto do velório do menino e ai as pessoas criticaram bastante e eu tirei a foto, mas eu achei que assim eu estava expressando a minha reivindicação, não foi legal..." (GER07, 35 anos, Diretora de Negócios)

Dentre as características comportamentais positivas, foi resultante do grupo de entrevistados em grande parte a manifestação de conquistas, realizações pessoais e profissionais, cujo principal objetivo era marcar um acontecimento importante. Houve aqueles que também utilizaram publicações com viés promocional, a fim de se fazer conhecer suas propostas. Os entrevistados obtiveram êxito não esperado, mas que ainda assim estavam dentro de um escopo condizente a situação, de modo que agregou um reconhecimento ou estabelecimento de contato de forma positiva. Alguns desdobramentos estão apresentados no quadro 6 .

Em atenção ao aspecto negativo, notou-se que a maioria dos comportamentos manifestados na rede, foram oriundos de publicações de terceiros, que por vez contribuíram para uma repercussão negativa ao indivíduo. Houve também aqueles que foram compreendidos sob a ótica negativa, apenas por ter seu juízo 
de valor não percebido pela sociedade da mesma forma que fora concebido e planejado pelo gestor. Os entrevistados utilizam algumas ferramentas de gestão de perfil, propostas pela RSO Facebook, porém muitos não conhecem em sua totalidade, ou até mesmo não dispõem de tempo disponível para configurá-las de modo a agir em seu favor.

\section{DISCUSSÃO}

O objetivo desse estudo foi compreender como gestores planejam seu comportamento nas RSO, em específico no Facebook. Os resultados mostraram que o modelo teórico de Ajzen (1991) aplica-se de forma significativa a explicação do comportamento planejado dos gestores, desenvolvido pela amostra de entrevistados. $O$ modelo que até então fora raramente utilizado na literatura para explicar o comportamento planejado dos gestores nas RSO, mostrou-se alinhado ao mesmo, com base nos resultados obtidos com a pesquisa.

Os dados analisados sugerem que os gestores, ao adotarem um perfil na RSO Facebook, o fazem sempre com algum objetivo específico, mesmo que num primeiro momento seja para cunho pessoal, como é a proposta da rede Facebook e tende a desencadear um elo com o círculo profissional do gestor. 0 ambiente virtual dessa massiva rede de relacionamentos é utilizado geralmente de forma inadequada, por grande parte dos usuários, dado que nem sempre os mesmos conhecem ou sabem utilizar todas as suas configurações.

Notou-se que os gestores têm dificuldades, com base na amostra estudada, em gerenciar essas configurações para um melhor direcionamento da manifestação de suas ações na rede Facebook. Percebeuse que esses gestores planejam o seu comportamento, muitas das vezes sem levar em consideração os seus valores, os quais segundo o modelo teórico estão atrelados às normas subjetivas do indivíduo.

As características referentes às normas subjetivas do indivíduo, segundo a proposta de Ajzen (1991) é um dos componentes no processo de formação da intenção do indivíduo, a ser manifestada em seu comportamento, assim como a percepção de atitudes relacionadas ao comportamento e ao controle do comportamento percebido. Na amostra estudada, identificou-se que as normas referentes aos indivíduos tendem a ser ocultadas pelo controle do comportamento percebido, dado a significância do cargo ocupado por esse gestor.

Com base na amostra estudada, os principais comportamentos manifestados por esses gestores são influenciados pelas consequências oriundas de seu comportamento, sejam essas questões positivas ou negativas. Quando tais consequências não são anteriormente medidas e tornam-se negativas, as ações corretivas são tomadas com base no controle do comportamento percebido.

A atitude relacionada ao comportamento é levada em consideração por esse gestor, com base na amostra estudada, para orientação pessoal e de sua equipe, sobre ações que não trazem resultados positivos. Os entrevistados não apresentaram nenhum tipo de aproveitamento comportamental, com base nas ações de outros indivíduos presentes em sua rede. 


\section{CONCLUSÕES}

Com base nos estudos anteriores (BAKER et al., 2010; GOLWAL et al., 2012; O'BRIEN et al., 2012; PIMMER et al., 2012; NADKARNI et al., 2012, OLLIER-MALATERRE et al., 2012), a pesquisa contribui para o processo de compreensão do comportamento planejado dos gestores na RSO Facebook, utilizando como modelo teórico para essa explicação, a TCP, que até então não fora localizada em estudos anteriores como modelo teórico para explicar o objetivo dessa pesquisa. A principal observação recai sobre a ação do controle do comportamento percebido, como agente moderador e corretor das ações de comportamento planejado dos gestores. Essa variável age diretamente sobre as suas intenções antes de manifestar o seu comportamento planejado, como ação moderadora e, após a manifestação do comportamento planejado como agente corretivo, dado que o desdobramento de sua ação tenha tido consequência negativa.

A significância atribuída ao cargo do gestor foi analisada, com base nas entrevistas e sob a ótica do comportamento planejado, como característica complementar a ação do controle do comportamento percebido. Os gestores têm um maior cuidado com a sua imagem refletida em suas ações comportamentais na RSO Facebook, considerando que, no ambiente profissional, os mesmo ocupam cargos que são observados por sua equipe, demais colegas dentro da organização, colegas de profissão, superiores diretos e indiretos e também em seu ambiente pessoal. Estudos futuros podem comparar diferenças de comportamento de gestores de setores diferentes, idade e/ou gênero distintos, ou ainda, daqueles gestores lotados em grupos de minorias, considerando a miscigenação brasileira, por exemplo, negros, lésbicas, homossexuais e outros.

Para uma maior capacidade preditiva do modelo, face à compreensão do comportamento planejado, recomenda-se também uma ampliação das RSO estudadas e a inserção da identificação de valores presente nesses gestores, para maior precisão em relação às variáveis que agem em forma conjunta para transformação de uma ou mais intenções em comportamentos manifestados de forma planejada.

\section{REFERÊNCIAS}

AJZEN, I.. The theory of planned behavior. Organizational Behavior and Human Decision Processes, v.50, n.2, p.179211, 1991.

AL-DEBEI, MUTAZ, M.; AL-LOZI, E.; PAPAZAFEIROPOULOU, A.. Why people keep coming back to Facebook: Explaining and predicting continuance participation from an extended theory of planned behavior perspective. Decision Support Systems, v.55, n.1, p.43-54, 2013.

BAKER, R. K., WHITE, K. M.. Predicting adolescents' use of social networking sites from an extended theory of planned behavior perspective. Computers in Human Behavior, v.26, n.6, p.1591-1597, 2010.

BARDIN, L.. L'Analyse de Contenu. Paris: Presses Universitaires de France, 1977.

BITNER, M. J.; BOOMS, B. H.; TETREAULT, M. S.. The service encounter: diagnosing favorable and unfavorable incidents. Journal of Marketing, v.54, n.1, p.71-84, 1990.
CHAFFEY, D.; ELLIS-CHADWICK, F.; MAYER, R.; JOHNSTON, K.. Internet Marketing: Strategy, implementation and practice. 4 ed. Reino Unido: Pearson Education Limited, 2009.

CÔTÉ, F.; GAGNON, J.; HOUME, P.; ABDELJELIL, A.; GAGNON, M.. Using The Theory of Planned Behaviour to predict nurses' intention to integrate research evidence into clinical decision-making. Journal of Advanced Nursing, v.68, n.10, p.2289-2298, 2012.

DARVELL, M. J.; WALSH, S. P.; WHITE, K. M.. Facebook tells me so: applying the theory of planned behavior to understand partner-monitoring behavior on facebook. Cyberpsychology, Behavior, and Social Networking, v.14, n.12, p.717-722, 2011.

FLANAGAN, J.. A técnica do incidente crítico. Arquivo Brasileiro de Psicologia Aplicada, v.25, n.2, p.99-141, 1973.

GOLWAL, M. D.; KALBANDE, D. T.; SONWANE, S. S. Profissionais da informação e o papel do facebook: consciência sobre sua utilidade no âmbito das redes sociais. 
Brazilian Journal of Information Science, v.6, n.1, p.85-100, 2012.

GREMLER, D. D.. The Critical Incident Technique in Service Research. Journal of Service Research, v.7, n.1, p.65-89, 2004.

GROSS, R.; ACQUISTI, A.. Information revelation and privacy in online social networks. In: WORKSHOP ON PRIVACY IN THE ELETRONIC SOCIETY, 05. Anais. Alexandria: 2005.

HSU, M. C.. The management of sports tourism: a causal modeling test of the Theory of Planned Behaviour. International Journal of Management, v.30, n.2, p.474-491, 2013.

HWANG, H. J.. Benefits and Challenges of Social Networks in Kazakhstan. International Journal of Software Engineering and Its Applications, v.7, n.4, p.415-426, 2013.

JOHNSTON, R.. The determinants of servisse quality: satisfiers and dissatisfiers. International Journal of Service Industry Management, v.6, n.5, p.53-71, 1995.

KWON, O.; WEN, Y.. An empirical study of the factors affecting social network service use. Computers in Human Behavior, v.26, n.2, p.254-263, 2010.

LAMPE, C.; ELLISON, N.; STEINFIELD, C.. A Face(book) in the crowd: social searching vs. social browsing. In: ANNIVERSARY CONFERENCE ON COMPUTER SUPPORTED COOPERATIVE WORK, 20. Anais. New York: ACM Press, 2006.

MCEACHERN, R. W.. Experiencing a social network in an organizational context: the facebook internship, Business Communication Quarterly, v.74, n.4, p.486-493, 2011.

NADKARNI, A.; HOFMANN, S. G.. Why do people use Facebook?. Personality and Individual Differences, v.52, n.3, p.243-249, 2012.

O'BRIEN, D.; TORRES, A. M.. Social networking and online privacy: Facebook users' perceptions. The Irish journal of management, v.31, n.2, p.63-97, 2012.

OLLIER-MALATERRE, A.; ROTHBARD, N. P.; BERG, J.. When worlds collide in cyberspace: how boundary work in online social networks impacts professional relationships. Academy of Management Review, v.38, n.4, p.645-669, 2013.

PARK, H. S.; WOOK, L. D.. A Test of Theory of Planned Behavior In Korea: Participation In Alcohol-Related Social Gatherings. International Journal of Psychology, v.44, n.6, p.418-433, 2013.
PELLING, E. L.; WHITE, K. M.. The theory of planned behavior applied to young people's use of social networking web sites. Cyberpsychology\& Behavior, v.12, n.6, p.755-759, 2009.

PERRY, A. R.; LANGLEY, C.. Even with the best of intentions: paternal involvement and the Theory of Planned Behavior. Family Process, v.52, n.2, p.179-192, 2013.

PICKETT, L. L.; GINSBURG, H. J.; MENDEZ, R. V.; LIM, D. E.; BLANKENSHIP, K. R.; FOSTER, L. E.; LEWIS, D. H.; RAMON, S. W.; SALTIS, B. M.; SHEFFIELD, S. B.. Ajzen's Theory of planned behavior as it relates to eating disorders and body satisfaction. North American Journal Of Psychology, v.14, n.2, p.339-354, 2013.

PIMMER, C.; LINXEN, S.; GRÖHBIEL, U.. Facebook as a learning tool? A case study on the appropriation of social network sites from mobile phones in developing countries. British Journal of Educational Technology, v.43, n.5, p.726738, 2012.

RANIERI, M.; MANCA, S.; FINI, A.. Why (and how) do teachers engage in social networks? an exploratory study of professional use of facebook and its implications for lifelong learning. British Journal of Educational Technology, v.43, n.5, p.754-769, 2012.

ROSENBLUM, D.. What anyone can know: the privacy risks of social networking sites, IEEE Security and Privacy, v.5, n.3, p.40-49, 2007.

STUTZMAN, F.. An evaluation of identity-sharing behavior in social network communities. Chapel Hill: The University of North Carolina at Chapel Hill, 2006.

TURVEY, K.. Questioning the character and significance of convergence between social network and professional practices in teacher education. British Journal of Educational Technology, v.43, n.5, p.739-753, 2012.

WILCOX, K.; STEPHEN, A. T.. Are close friends the enemy? Online social networks, self-esteem, and self-control. Journal of Consumer Research, v.40, n.1, p.90-103, 2013.

XIAO, J. J.; TANG, C.; SERIDO, J.; SHIM, S.. Antecedents and consequences of risky credit behavior among college students: application and extension of the theory of planned behavior. Journal Of Public Policy \& Marketing v.30, n.2, p.239-245, 2011.

ZAKARIA, M. H.; WATSON, J.; EDWARDS, S. L.. Investigating the use of web 2.0 technology by Malayasin students. Multicultural Education and Technology Journal, v.4, n.1, p.12-29, 2010 